Review began 08/24/2021 Review ended 09/14/2021 Published 09/18/2021

๑) Copyright 2021

Fujita-Yamashita et al. This is an open access article distributed under the terms of the Creative Commons Attribution License CC-BY 4.0., which permits unrestricted use, distribution, and reproduction in any medium, provided the original author and source are credited.

\section{Gender-Dependent Characteristics of Serum 1,25- Dihydroxyvitamin D/25-Hydroxyvitamin D Ratio for the Assessment of Bone Metabolism}

\author{
Manami Fujita-Yamashita ${ }^{1}$, Koichiro Yamamoto ${ }^{1}$, Hiroyuki Honda ${ }^{1}$, Yoshihisa Hanayama ${ }^{1}$, Kazuki \\ Tokumasu $^{1}$, Yasuhiro Nakano ${ }^{1}$, Kou Hasegawa ${ }^{1}$, Hideharu Hagiya ${ }^{1}$, Mikako Obika ${ }^{1}$, Hiroko Ogawa ${ }^{1}$, \\ Fumio Otsuka ${ }^{1}$ \\ 1. Department of General Medicine, Okayama University Graduate School of Medicine, Dentistry and Pharmaceutical \\ Sciences, Okayama, JPN
}

Corresponding author: Fumio Otsuka, fumiotsu@md.okayama-u.ac.jp

\section{Abstract \\ Objectives}

Vitamin D deficiency, which is common worldwide, increases the risks of falls and fractures and can lead to increased morbidity and mortality. However, the clinical utility and relevance of vitamin D activation remain unknown. The aim of the present study was to clarify the clinical usefulness of serum 1,25-dihydroxyvitamin $\mathrm{D}(1,25 \mathrm{D}) / 25$-hydroxyvitamin $\mathrm{D}$ (25D) ratio for assessment of the extent of bone metabolism.

\section{Methods}

We retrospectively screened data for 87 patients whose serum 1,25D and 25D levels were measured. Eight patients who were taking vitamin D preparations were excluded, and data for 79 patients (33 males and 46 females) were analyzed. Since menopausal status can be associated with serum vitamin D level, we divided the patients by gender and divided the female patients into two groups at the age of 50 years.

\section{Results}

The median serum 1,25D/25D ratio was significantly lower in males than in females, with the most considerable difference in all males [4.1 (interquartile range: $2.3-5.8) \times 10^{-3}$ ] versus elderly females (aged $\geqq 50$ years) $\left[7.9(3.3-10.1) \times 10^{-3}\right)$. Main disorders were endocrine (30.6\%), inflammatory (18.5\%), and bonerelated (16.7\%) disorders. The ratios of serum $1,25 \mathrm{D} / 25 \mathrm{D}$ had significant negative correlations with femoral dual-energy X-ray absorptiometry \% young adult mean (DEXA \%YAM) $(R=-0.35)$ and lumbar DEXA \%YAM $(R=-0.32)$. Significant correlations were found between the $1,25 \mathrm{D} / 25 \mathrm{D}$ ratio and serum levels of inorganic phosphate (iP), parathyroid hormone, and alkaline phosphatase (ALP). The 1,25D/25D ratio had genderspecific characteristics: the ratio was significantly correlated with age in males $(R=-0.49)$, while it was significantly correlated with BMI in females $(R=0.34)$.

\section{Conclusions}

The results of this study suggested that vitamin D activity is negatively correlated with bone mineral density, being reduced in aged males but enhanced in obese females.

Categories: Endocrinology/Diabetes/Metabolism, Internal Medicine

Keywords: aging, bone metabolism, calcium, cyp27b1, vitamin d

\section{Introduction}

Vitamin $\mathrm{D}$ is obtained in the body by food intake or by production from 7-dehydrocholesterol by exposure of the skin to ultraviolet B radiation [1]. Vitamin D is first metabolized in the liver to 25-hydroxyvitamin D (25D), which is a major circulating metabolite [1]. In the kidney, 25D is subsequently metabolized to the hormonally active form, 1,25-dihydroxyvitamin D (1,25D), via $1 \alpha$-hydroxylase encoded by the CYP27B1 gene [1]. Renal CYP27B1 is regulated primarily by parathyroid hormone (PTH) and calcitonin in stimulatory manners and by fibroblast growth factor-23 (FGF-23) and 1,25D itself in inhibitory manners [1]. CYP27B1 also exists in extra-renal sites such as macrophages, and its expression in extra-renal sites is associated with granuloma-forming disorders and is regulated mainly by type I and type II interferons (IFNs) [2]. 1,25D has a cellular effect through the vitamin D receptor (VDR) [3], which leads to calcium absorption in the gut, bone metabolism, and parathyroid function.

Serum 25D level has been considered to be a reliable marker of vitamin D status: serum 25D level below 20 $\mathrm{ng} / \mathrm{mL}$ is defined as vitamin D deficiency and serum 25D level below $30 \mathrm{ng} / \mathrm{mL}$ is defined as vitamin D insufficiency [4]. A recent study showed that a low vitamin D status is common worldwide and is associated 
with various diseases including kidney, heart, and liver failure, secondary hyperparathyroidism, osteomalacia, inflammatory bowel disease, granuloma-forming disorders (sarcoidosis and tuberculosis), and cancer [5]. Vitamin D deficiency also increases the risks of falls, fractures, bone loss, and sarcopenia [6-8], leading to worse outcomes of illness severity, morbidity, and mortality [9-11]. However, since the evaluation of only serum 25D level did not explain hormonal activity of vitamin D, it is conceivable that we should take serum 1,25D level into account as well. The clinical utility of and the relevance to pathophysiology of evaluation of the ratio $1,25 \mathrm{D} / 25 \mathrm{D}$, which indicates the extent of vitamin $\mathrm{D}$ activation, have remained unknown.

In the present study, we retrospectively investigated the relevance of activation of vitamin $\mathrm{D}$ to various clinical characteristics of patients who visited a general medicine department.

\section{Materials And Methods}

\section{Study design}

We conducted a single-center cross-sectional study: the medical records of 87 patients (male/female: $35 / 52$ ), whose serum levels of 25D and 1,25D were measured between January 2017 and December 2019 at the Department of General Medicine, Okayama University Hospital were screened. Of those patients, 8 patients ( 2 males and 6 females) who were taking vitamin D preparations were excluded, and data for 79 patients (33 males (41.8\%) and 46 females (58.2\%)) were analyzed. The decision to examine serum levels of 25D or 1,25D had been made individually by physicians for clinical purposes when vitamin D-related disorders such as hyper- and hypoparathyroidism, osteoporosis, and granuloma-forming disorders were suspected [5]. Data for other biochemical parameters were obtained within one week from the measurement of 25D or 1,25D. The present study was approved by the Ethical Committee of Okayama University Hospital (KEN2001-022) and adhered to the Declaration of Helsinki.

\section{Analysis of clinical parameters}

Information on the patients' main disorders and past medical histories was obtained from hospital medical records. Information on age, gender, race, body mass index (BMI), and self-rating depression scale (SDS) was also obtained [12]. Information on the following biochemical parameters was also obtained: white blood cells, red blood cells, hemoglobin, hematocrit and platelets for blood cell counts; 25D, 1,25D, calcium (Ca), corrected $\mathrm{Ca}(\mathrm{cCa})$, inorganic phosphate (iP), $\mathrm{cCa} \times \mathrm{iP}$, alkaline phosphatase (ALP), bone-specific alkaline phosphatase (BAP), and intact PTH for bone metabolism; total bilirubin, total protein, albumin, aspartate aminotransferase (AST), alanine aminotransferase (ALT), lactate dehydrogenase (LDH), $\gamma$-glutamyl transpeptidase ( $\mathrm{YGTP}$ ), sodium, potassium, chloride, magnesium, blood urea nitrogen (BUN), creatinine and estimated glomerular filtration rate (eGFR) for liver and renal functions; prothorombin time-international normalized ratio (PT-INR), activated partial thromboplastin time (APTT), and d-dimer for coagulatory markers; C-reactive protein (CRP), erythrocyte sedimentation rate in one hour (ESR), ferritin, $50 \%$ hemolytic unit of complement (CH50) and angiotensin-converting enzyme (ACE) for inflammatory markers; and hemoglobin A1c (HbA1c), plasma glucose, total cholesterol, uric acid, adrenocorticotropic hormone (ACTH), cortisol, prolactin, luteinizing hormone (LH), follicle-stimulating hormone (FSH), growth hormone (GH), thyroid-stimulating hormone (TSH), free thyroxine (FT4), ratio of TSH/FT4, and total testosterone for endocrine and metabolic markers. The levels of those parameters were determined by using an autoanalyzer system at the Central Laboratory of Okayama University Hospital. The levels of 25D and 1,25D were determined by a radioimmunoassay and a chemiluminescent immunoassay, respectively, at LSI Medience Corporation (Tokyo). Radiological data of dual-energy X-ray absorptiometry \% young adult mean (DEXA \%YAM) in the femoral bone and lumbar spine were evaluated as we previously reported [13].

\section{Statistical analysis}

All statistical analyses were performed using EZR, version 1.40 (Saitama Medical Center, Jichi Medical University, Saitama, Japan), which is a graphical user interface for R (The R Foundation for Statistical Computing, Vienna, Austria) [14]. In more detail, it is a modified version from R commander designed to add functions of frequently used statistics in biostatistics. Continuous measurements were statistically tested using the Mann-Whitney U test, Spearman's rank correlation coefficient, or Kruskal-Wallis test. The MannWhitney U test and Spearman's rank correlation coefficient were treated as two-sided. When differences were detected by the Kruskal-Wallis test, the Steel-Dwass post-hoc test was used for further analysis between the groups. $P$-values less than 0.05 were regarded as statistically significant. There were potential confounding factors: diseases such as hyperparathyroidism, sarcoidosis, inflammatory bowel disease, osteoporosis, and osteomalacia or rickets; environmental factors such as seasonal changes, lifestyles related to sunlight exposure, and nutritional intake; and gender and the human race. Since menopause has been reported to cause changes in serum vitamin D level and bone metabolism [15], we conducted stratified analysis by gender and we divided the female patients into two groups at the age of 50 years [16].

\section{Results}

Patients' characteristics and relations to vitamin D activity 
The 79 patients analyzed in the present study included 33 males (41.8\%) and 46 females (58.2\%). As shown in Figure $1 A$, the median age of the male patients was 65 [interquartile range (IQR): 45-74] years and that of the female patients was 61.5 (IQR: 40.8-71.8) years, and there was no significant difference in the median ages. The median BMI of males was 21.7 (IQR: 18.6-24.1) kg/m² and that of females was 22.0 (IQR: 18.9$24.5) \mathrm{kg} / \mathrm{m}^{2}$, and the difference was not significant (Figure $1 B$ ). The median serum 25D level in males was 12.5 (IQR: 8.4-18.6) ng/mL and that in females was 10 (IQR: 7.5-14.5) ng/mL (Figure 1C). The median serum 1,25D level in males was 47 (IQR: 41.0-64.3) pg/mL and that in females was 58 (IQR: 41.0-83.0) pg/mL (Figure $1 D$ ). There was no significant difference between males and females in the level of 25D (Figure 1C) or 1,25D (Figure 1D), but the median serum 1,25D/25D ratio was significantly lower in males than in females: the median ratio in males was $4.1(\mathrm{IQR}: 2.3-5.8) \times 10^{-3}$ and that in females was 6.8 (IQR: 3.0-9.8) $\times$ $10^{-3}$ (Figure 1E). Regarding electrolytes, serum cCa, iP, and cCa $\times$ iP levels were not significantly different in males and females (Figure $1 F-1 H$ ). The median serum levels of cCa were 9.5 (IQR: 9.1-9.9) mg/dL in males and 9.5 (IQR: 9.2-10.3) $\mathrm{mg} / \mathrm{mL}$ in females, those of iP were 3.4 (IQR: $2.9-3.9) \mathrm{mg} / \mathrm{dL}$ in males and 3.5 (IQR: $2.8-4.0) \mathrm{mg} / \mathrm{dL}$ in females, and those of cCa $\times \mathrm{iP}$ were $31.5(\mathrm{IQR}: 28.6-36.5)(\mathrm{mg} / \mathrm{dL})^{2}$ in males and 33.3 (IQR: 29.5-38.4) $(\mathrm{mg} / \mathrm{dL})^{2}$ in females.
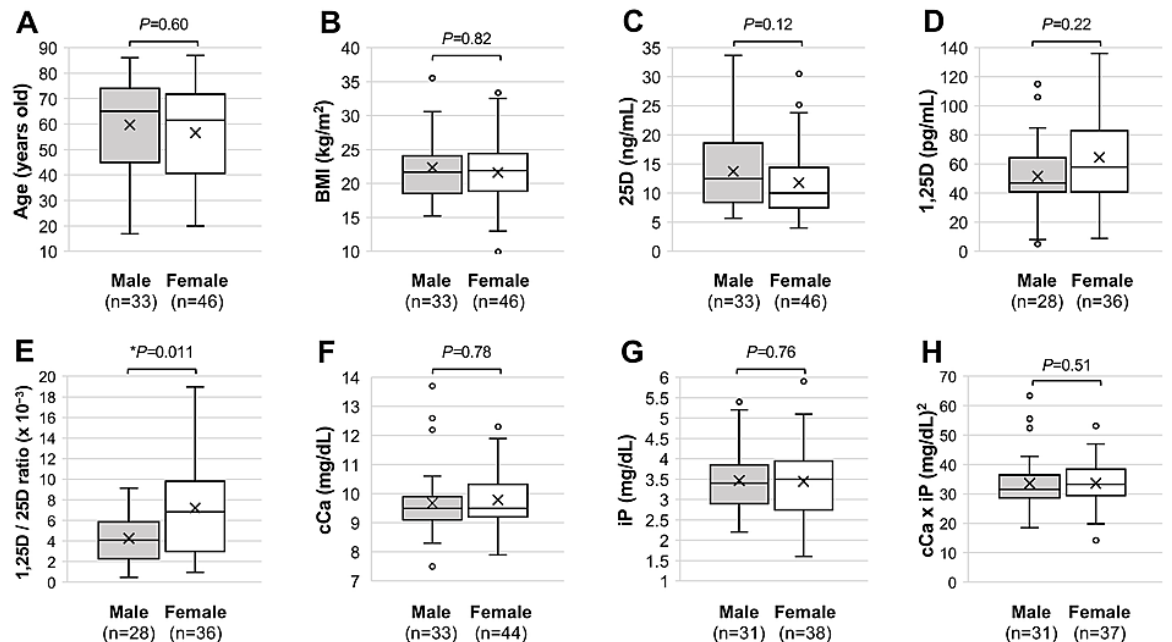

$\underset{(n=31)}{\operatorname{Male}} \underset{(\mathrm{n}=37)}{\text { Female }}$

\section{FIGURE 1: Characteristics of the male and female patients}

Age (A), BMI (B) and serum levels of 25D (C), 1,25D (D), 1,25D/25D ratio (E), cCa (F), iP (G), and cCa $\times$ iP $(H)$ are shown. In each panel, the upper horizontal line, lower horizontal line, and horizontal bar of the box indicate the 75 th percentile, 25 th percentile, and median, respectively. The horizontal bars outside the box are the maximum and minimum values within 1.5 times the interquartile range. The $X$ sign within the box indicates the mean. ${ }^{*} P<0.05$, a statistically significant difference between the indicated groups $(\mathrm{A}-\mathrm{H})$. BMI: body mass index; cCa: corrected calcium; iP: inorganic phosphate; n.s.: not significant; 1,25D: 1,25-dihydroxyvitamin D; 25D: 25hydroxyvitamin D.

The patients' main disorders and past medical histories are shown in Table 1. The most frequent main disorders were endocrine disorders (30.6\%, 33 of 108 disorders) including primary hyperparathyroidism $(10.2 \%, 11 / 108)$ and ectopic hyperparathyroidism $(2.9 \%, 2 / 108)$ for vitamin D-related disorders followed by inflammatory disorders $(18.5 \%, 20 / 108)$ including sarcoidosis $(2.8 \%, 3 / 108)$ and inflammatory bowel disease $(2.8 \%, 3 / 108)$ and bone-related disorders including osteoporosis $(13.9 \%, 15 / 108)$ and osteomalacia or rickets $(2.8 \%, 3 / 108)$ (Table 1 , left). The most frequent past medical history was malignancy $(26.8 \%, 19$ of 71 histories) followed by bone-related diseases $(16.9 \%, 12 / 71)$ including fractures $(11.3 \%, 8 / 71)$ and osteoporosis $(5.6 \%, 4 / 71)$, metabolic diseases $(14.1 \%, 10 / 71)$, and ureterolithiasis $(11.3 \%, 8 / 71)$ (Table 1 , right). Past medical histories possibly related to vitamin D deficiency included sarcoidosis $(1.4 \%, 1 / 71)$ in inflammatory diseases $(8.5 \%, 6 / 71)$, while there was no past history of hyper- or hypoparathyroidism (Table 1 , right). 


\section{Cureus}

\begin{tabular}{|llll}
\hline Main disorders & Past medical histories & Total (\%) \\
Categories & Total $(\%)$ & Categories & $19(26.8)$ \\
Endocrine disorder & $33(30.6)$ & Malignancy & $12(16.9)$ \\
Inflammatory disorder & $20(18.5)$ & Bone-related disease & $10(14.1)$ \\
Bone-related disorder & $18(16.7)$ & Metabolic disease & $8(11.3)$ \\
Mental disorders & $10(9.3)$ & Ureterolithiasis & $6(8.5)$ \\
Gastroenterological & $9(8.3)$ & Inflammatory disease & $5(7.0)$ \\
Bacterial infection & $6(5.6)$ & CNS-related disease & $5(7.0)$ \\
Haematological & $4(3.7)$ & Mental disease & $4(5.6)$ \\
Malignancy & $2(1.9)$ & Endocrine disease & $2(2.8)$ \\
Other & $6(5.6)$ & Other & $71(100)$ \\
Total & $108(100)$ & Total &
\end{tabular}

TABLE 1: Main disorders and past medical histories

CNS: central nervous system.

\section{Gender-dependent differences of vitamin D activity and bone mineral density}

Since menopausal status has been reported to be associated with vitamin D and bone metabolism [15], we divided the female patients into two groups at the age of 50 years [16]. Serum 1,25D/25D ratios were found to be significantly higher in female patients aged $\geqq 50$ years (median ratio, 7.9 (IQR: $3.3-10.1) \times 10^{-3}$ ) than in male patients (median ratio, 4.1 (IQR: 2.3-5.8) $\times 10^{-3}$ ) (Figure $2 \mathrm{~A}$ ). Regarding bone mineral density, female patients aged $\geqq 50$ years had significantly lower values of DEXA \%YAM in both the femoral bone (Figure $2 B$ ) and lumbar spine (Figure 2C) than those in male patients. The median values of DEXA \%YAM in male patients were $84 \%$ (IQR: 74-92\%) in the femoral bone and 98\% (IQR: $83.5-108 \%$ ) in the lumbar spine, and those in female patients aged $\geqq 50$ years were $63 \%$ (IQR: $55.8-72.8 \%$ ) in the femoral bone and $72 \%$ (IQR: 64 $87 \%$ ) in the lumbar spine.
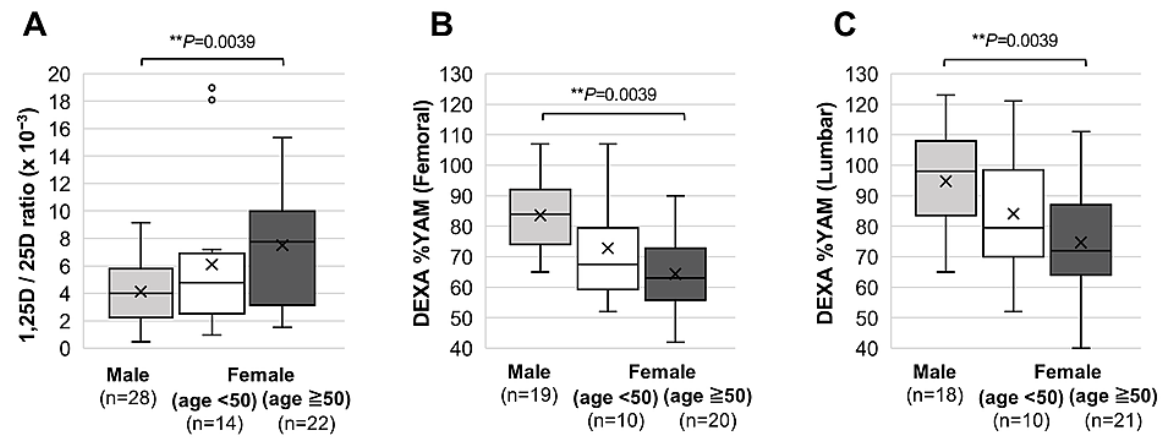

FIGURE 2: Gender-dependent differences of serum 1,25D/25D ratio and bone mineral densities

Serum 1,25D/25D ratio $(A)$ and bone mineral densities in the femoral bone $(B)$ and lumbar spine $(C)$ were compared between male patients, female patients aged $<50$ years, and female patients aged $\geqq 50$ years. The details of each panel are shown in the legend of Figure $1 .{ }^{* *} P<0.01$ and ${ }^{*} P<0.05$, statistically significant correlations between the indicated factors. 1,25D: 1,25-dihydroxyvitamin D; 25D: 25-hydroxyvitamin D; DEXA $\%$ YAM: dual-energy $\mathrm{X}$-ray absorptiometry $\%$ young adult mean.

Relationships of serum 1,25D/25D ratios with clinical markers in bone 


\section{Cureus}

\section{metabolism}

We investigated the correlations of serum $1,25 \mathrm{D} / 25 \mathrm{D}$ ratios with various clinical parameters. Of note, as shown in Figure 3, 1,25D/25D ratios had significant negative correlations with bone mineral densities including femoral DEXA \%YAM $\left(R=-0.35,{ }^{*} P<0.05\right.$; Figure $\left.3 A\right)$ and lumbar DEXA \%YAM $(R=-0.32$, * $P<0.05$; Figure $3 B$ ). Regarding biochemical markers of bone metabolism, $1,25 \mathrm{D} / 25 \mathrm{D}$ ratios were not correlated with serum albumin $(R=0.19, P=0.13$; Figure $4 A)$ and $\mathrm{cCa}(R=0.17, P=0.18$; Figure $4 B)$ levels. On the other hand, $1,25 \mathrm{D} / 25 \mathrm{D}$ ratios had significant positive correlations with serum levels of iP $(R=-0.34$, *** $P<0.01$; Figure $4 C)$, intact PTH $\left(R=0.64,{ }^{* * *} P<0.01\right.$; Figure $\left.4 D\right)$, ALP $\left(R=0.46,{ }^{*} P<0.05\right.$; Figure $\left.4 E\right)$, and BAP $(R=0.62, * * P<0.01$; Figure $4 F) .1,25 \mathrm{D} / 25 \mathrm{D}$ ratios had significant negative correlations with creatinine $\left(R=-0.26,{ }^{*} P<0.05\right.$; Figure $\left.4 G\right)$ and FT4 $\left(R=-0.28,{ }^{*} P<0.05\right.$; Figure $\left.4 H\right)$. The correlations of $1,25 \mathrm{D} / 25 \mathrm{D}$ ratios with other clinical parameters are summarized in Table 2 . There were no significant correlations of 1,25D/25D with SDS of the patients' profile, blood cell count, liver function, coagulatory markers, inflammatory markers, and endocrine and metabolic markers (Table 2).

A

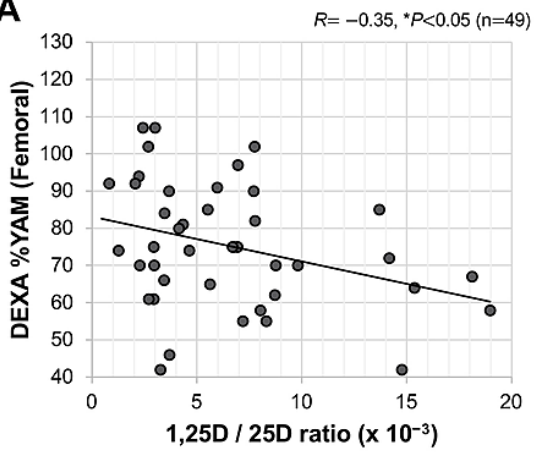

B

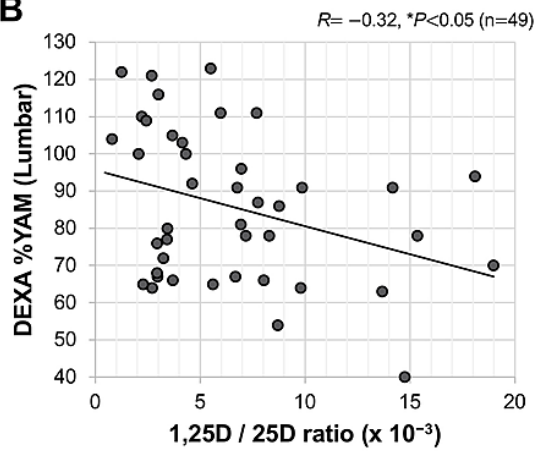

FIGURE 3: Correlations of serum 1,25D/25D ratio with bone mineral densities

Correlations of femoral DEXA \%YAM (A) and lumbar DEXA \%YAM (B) with a 1,25D/25D ratio in all patients are shown. ${ }^{*} P<0.05$, statistically significant correlations between the indicated factors (A, B). 1,25D: 1,25dihydroxyvitamin D; 25D: 25-hydroxyvitamin D; DEXA \%YAM: dual-energy X-ray absorptiometry \% young adult mean. 


\section{Cureus}

A
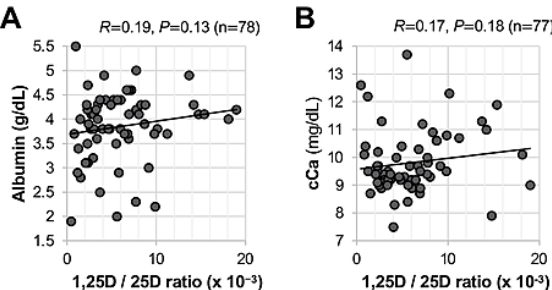

E $R=0.46,{ }^{*} p<0.05(n=36)$

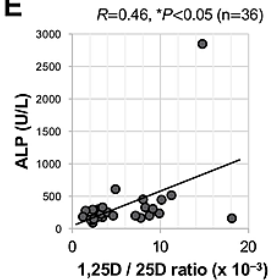

F $\quad R=0.62, * p<0.01(n=17)$

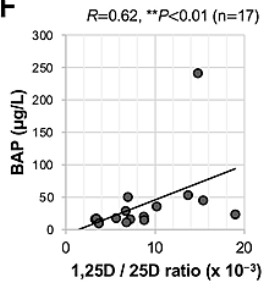

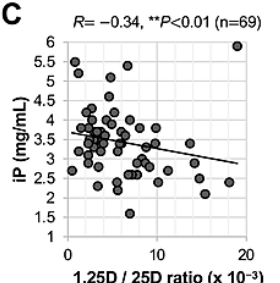

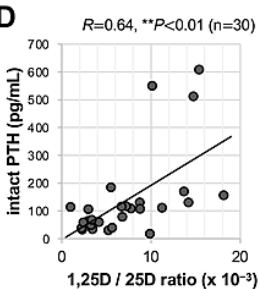

G $R=-0.26,{ }^{*} p<0.05(n=79)$

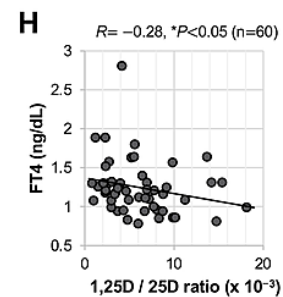

FIGURE 4: Relationships of 1,25D/25D ratio with bone metabolism and clinical characteristics

Correlations of serum 1,25D/25D ratios with albumin (A), cCa (B), iP (C), intact PTH (D), ALP (E), BAP (F), creatinine $(\mathrm{G})$, and FT4 $(\mathrm{H})$ are shown. ${ }^{* *} P<0.01$ and ${ }^{*} P<0.05$, statistically significant correlations between the indicated factors. ALP: alkaline phosphatase; BAP: bone-specific alkaline phosphatase; cCa: corrected calcium; FT4: free thyroxine; iP: inorganic phosphate; n.s.: not significant; 1,25D: 1,25-dihydroxyvitamin D; PTH: parathyroid hormone; 25D: 25-hydroxyvitamin D.

\section{Comparison}

Patients' profile

Age

BMI

SDS

Blood cell count

White blood cell

Red blood cell

Hemoglobin

Hematocrit

Platelet

Bone metabolism

$\mathrm{Ca}$

$\mathrm{cCa}$

iP

$\mathrm{cCa} \times \mathrm{iP}$

ALP

BAP

Intact PTH

\section{All patients with 1,25D/25D}

Number

R

$-0.10$

P-values

79

79

41

0.26

$-0.11$

*0.036

0.56

$-0.21$

0.094

78

0.16

0.21

0.098

0.45

0.16

0.22

$-0.12$

0.34

0.18

0.16

0.17

0.18

77

69

68

$-0.34$

**0.0081

$-0.24$

0.065

36

17

30

0.46

*0.012

0.62

*0.0098

0.64

${ }^{* *} 0.00029$

Liver and renal functions

Total bilirubin

Total protein
68

70
0.00027

0.059
1.0

0.66 


\section{Cureus}

\begin{tabular}{|c|c|c|c|}
\hline Albumin & 78 & 0.19 & 0.13 \\
\hline AST & 74 & -0.038 & 0.77 \\
\hline ALT & 76 & -0.046 & 0.72 \\
\hline LDH & 75 & -0.093 & 0.47 \\
\hline yGTP & 74 & -0.052 & 0.70 \\
\hline Sodium & 79 & 0.020 & 0.88 \\
\hline Potassium & 79 & 0.093 & 0.47 \\
\hline Chloride & 79 & 0.15 & 0.25 \\
\hline Magnesium & 57 & 0.27 & 0.063 \\
\hline BUN & 78 & -0.22 & 0.083 \\
\hline Creatinine & 79 & -0.26 & ${ }^{*} 0.036$ \\
\hline eGFR & 78 & 0.11 & 0.38 \\
\hline \multicolumn{4}{|l|}{ Coagulatory markers } \\
\hline PT-INR & 38 & -0.28 & 0.10 \\
\hline APTT & 37 & -0.24 & 0.16 \\
\hline D-dimer & 30 & -0.11 & 0.60 \\
\hline \multicolumn{4}{|l|}{ Inflammatory markers } \\
\hline CRP & 72 & -0.24 & 0.067 \\
\hline ESR & 29 & -0.073 & 0.73 \\
\hline Ferritin & 30 & -0.12 & 0.56 \\
\hline $\mathrm{CH} 50$ & 22 & -0.11 & 0.63 \\
\hline ACE & 27 & 0.055 & 0.79 \\
\hline \multicolumn{4}{|c|}{ Endocrine and metabolic markers } \\
\hline $\mathrm{HbA1c}$ & 48 & -0.089 & 0.58 \\
\hline Plasma glucose & 55 & 0.24 & 0.12 \\
\hline Total cholesterol & 61 & 0.16 & 0.26 \\
\hline Uric acid & 72 & -0.06 & 0.65 \\
\hline АСTH & 30 & -0.30 & 0.15 \\
\hline Cortisol & 31 & 0.077 & 0.72 \\
\hline PRL & 15 & -0.40 & 0.18 \\
\hline LH & 17 & 0.16 & 0.56 \\
\hline FSH & 18 & 0.28 & 0.28 \\
\hline $\mathrm{GH}$ & 18 & -0.28 & 0.29 \\
\hline TSH & 62 & -0.12 & 0.40 \\
\hline FT4 & 60 & -0.28 & ${ }^{*} 0.047$ \\
\hline TSH/FT4 & 60 & 0.042 & 0.77 \\
\hline \multicolumn{4}{|l|}{ Bone mineral density } \\
\hline DEXA \%YAM (Femoral) & 49 & -0.35 & ${ }^{*} 0.023$ \\
\hline DEXA \%YAM (Lumbar) & 49 & -0.32 & ${ }^{*} 0.039$ \\
\hline
\end{tabular}




\section{Cureus}

\section{TABLE 2: Correlations between serum 1,25D/25D ratio and clinical parameters in all patients}

ACE: angiotensin-converting enzyme; ACTH: adrenocorticotropic hormone; ALP: alkaline phosphatase; ALT: alanine aminotransferase; APTT: activated partial thromboplastin time; AST: aspartate aminotransferase; BAP: bone-specific alkaline phosphatase; BMI: body mass index; BUN: blood urea nitrogen; CH50: 50\% hemolytic unit of complement; Ca: calcium; cCa: corrected calcium; CRP: C-reactive protein; DEXA \%YAM: dual-energy X-ray absorptiometry $\%$ young adult mean; eGFR: creatinine and estimated glomerular filtration rate; ESR: erythrocyte sedimentation rate in one hour; FSH: follicle-stimulating hormone; FT4: free thyroxine; yGTP: y-glutamyl transpeptidase; GH: growth hormone; HbA1c: hemoglobin A1c; iP: inorganic phosphate; LDH: lactate dehydrogenase; LH: luteinizing hormone; PRL: prolactin; PTH: parathyroid hormone; PT-INR: prothrombin time-international normalized ratio; SDS: selfrating depression scale; TSH: thyroid-stimulating hormone; 25D: 25-hydroxyvitamin D; 1,25D: 1,25-dihydroxyvitamin D.

\section{Relevance of serum 1,25D/25D ratios to patients' age and BMI}

$1,25 \mathrm{D} / 25 \mathrm{D}$ ratio did not show a significant correlation with age in all patients $(R=-0.10, P=0.43$; Figure $5 A)$. However, when the patients were divided by gender, there was a significant correlation of $1,25 \mathrm{D} / 25 \mathrm{D}$ ratio with age in male patients $(R=-0.49$, *** $P<0.01$; Figure $5 B)$, but there was no significant correlation in female patients $(R=0.21, P=0.22$; Figure $5 C) .1,25 \mathrm{D} / 25 \mathrm{D}$ ratio showed a significant positive correlation with BMI in all patients $\left(R=0.26\right.$, ${ }^{*} P<0.05$; Figure $\left.5 D\right)$. When the patients were divided by gender, there was no significant correlation in male patients $(R=0.13, P=0.51$; Figure $5 E)$, but there was a significant correlation in female patients $\left(R=0.34,{ }^{*} P<0.05\right.$; Figure $\left.5 F\right)$.

A

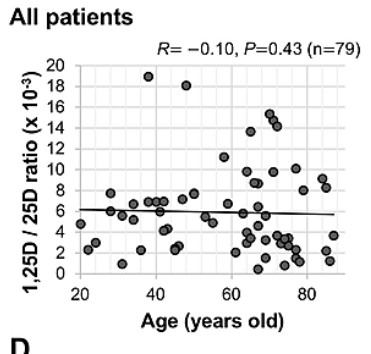

D

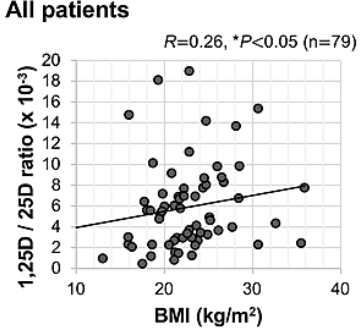

B

Male patients

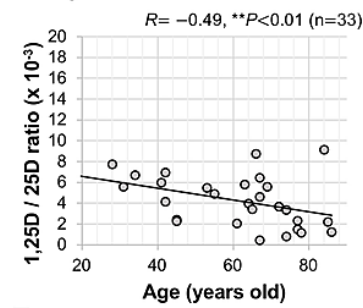

E

Male patients

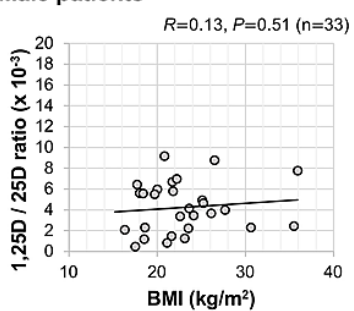

C

Female patients

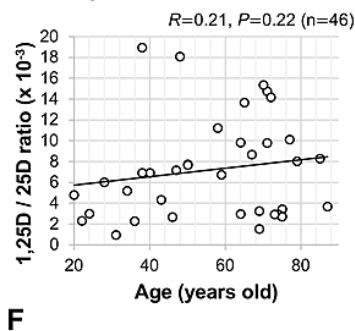

$\mathbf{F}$

Female patients

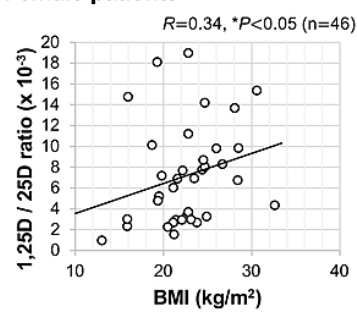

FIGURE 5: Correlations of patients' age and BMI in relation to serum $1,25 \mathrm{D} / 25 \mathrm{D}$ ratio

Correlations of age with serum 1,25D/25D ratio in all patients $(A)$, male patients $(B)$, and female patients $(C)$ are shown. Correlations of BMI with serum $1,25 \mathrm{D} / 25 \mathrm{D}$ ratio in all patients $(\mathrm{D})$, male patients $(\mathrm{E})$, and female patients (F) are also shown. ${ }^{* *} P<0.01$ and ${ }^{*} P<0.05$, statistically significant correlations between the indicated factors. BMI: body mass index; 1,25D: 1,25-dihydroxyvitamin D; 25D: 25-hydroxyvitamin D.

\section{Discussion}

The results of the present study suggested that vitamin D activities had relevance to clinical parameters, especially bone turnover, with gender-specific correlations with features in age and BMI. The ratio of serum $1,25 \mathrm{D} / 25 \mathrm{D}$ as a marker for activation of vitamin D was significantly lower in male patients than in female patients, particularly in older females ( $\geqq 50$ years of age), who are considered to be menopausal women. On the other hand, bone mineral density was significantly lower in older female patients ( $\geqq 50$ years of age) than in male patients. The serum $1,25 \mathrm{D} / 25 \mathrm{D}$ ratio was found to be negatively correlated with bone mineral density, negatively correlated with serum inorganic phosphate, and positively correlated with intact PTH, ALP, and BAP in all patients. Of interest, the ratio was negatively correlated with age in male patients but was positively correlated with BMI in female patients, suggesting that vitamin D activation is involved in bone metabolism in a gender-specific manner.

The 1,25D/25D ratio is a putative index of CYP27B1 activity and is considered to be a useful tool for the diagnosis of ocular sarcoidosis [17]. In cases of sarcoidosis or lymphomas, type II IFN enhances the activity of $1 \alpha$-hydroxylase in macrophages, resulting in increased production of $1,25 \mathrm{D}$ and 
hypercalcemia [1]. Excessive vitamin D activity also has a stimulatory effect on bone turnover and an inhibitory effect on bone mineralization [18]. Vitamin D is a key component of the bone-kidney-parathyroid endocrine axis. 1,25D produced in the kidney binds to VDR in the bone and also activates FGF-23 gene expression. Secreted FGF-23 acts on the Klotho-FGF receptor complex in the kidney and parathyroid gland. In the kidney, FGF-23 down-regulates the Cyp27b1 gene and up-regulates the Cyp24 gene, resulting in suppression of vitamin D activity. In the parathyroid gland, FGF-23 suppresses the expression of PTH, which has the potential function of promoting Cyp27b1 gene expression. Since there is a closed negative feedback loop for vitamin D homeostasis, disruption of the loop regulating CYP27B1 induction results in an increase in 1,25D level [19-21].

Vitamin D level in serum has been reported to decline with aging due to a reduction in the production of vitamin D in the skin [22,23]. In general, a hormonal decline of sex steroids such as androgen and estrogen is important in the aging process [24]. Total testosterone level has been reported to have a slight but significant positive association with serum 25D level, suggesting that both testosterone and vitamin D can be health-related markers for males [25]. A meta-analysis showed that vitamin D status has an inverse relationship with BMI in both diabetic and non-diabetic subjects [26]. Another meta-analysis showed that serum vitamin D level had an inverse association with the risk of abdominal obesity in a dose-response manner [27]. Vitamin D deficiency has been considered to be associated with obesity and metabolic dysregulation by modulating the expression of genes related to adipogenesis and inflammatory and oxidative stress in mature adipocytes [28].

In the present study, it was also shown that serum levels of creatinine and free thyroxin were negatively correlated with the serum $1,25 \mathrm{D} / 25 \mathrm{D}$ ratio. In this regard, patients with chronic kidney disease (CKD) usually have secondary hyperparathyroidism and a low serum 1,25 level [29]. Patients in an advanced stage of CKD have high levels of serum FGF-23 and PTH and a low level of Klotho expression, so-called FGF-23 resistance, leading to impaired activation of vitamin D [19]. Vitamin D also acts on the thyroid through VDR; however, there is no clear consensus about a relationship between vitamin D status and thyroid function in healthy humans [30], although a study on the role of vitamin D in thyroid diseases indicated that vitamin D deficiency might be an increased risk of autoimmune thyroid diseases [30]. However, based on the present findings, it seems likely that thyroid function is involved in the activation of vitamin D.

Vitamin D activity should be evaluated when vitamin D-related disorders such as hyperparathyroidism or granuloma-forming disorders are suspected. However, our findings presented here indicate the importance of assessing vitamin D activity from the ratio of $1,25 \mathrm{D}$ to $25 \mathrm{D}$ in general clinical settings. Considering that vitamin D activation can be linked to aging and obesity as well as bone mineral metabolism, measurement of serum $1,25 \mathrm{D} / 25 \mathrm{D}$ ratio can be useful for suspecting bone loss, fractures, sarcopenia, or other clinical outcomes associated with frailty. Since the present study showed a negative correlation between serum $1,25 \mathrm{D} / 25 \mathrm{D}$ ratio and bone mineral density, serum $1,25 \mathrm{D} / 25 \mathrm{D}$ ratio might be a marker for determining the necessity for vitamin D supplementation. However, when a high serum 1,25D/25D ratio is related to increased PTH as in primary hyperparathyroidism, vitamin D supplementation may promote the progression of hypercalcemia. Nevertheless, our findings suggest that a high serum $1,25 \mathrm{D} / 25 \mathrm{D}$ ratio is a clue for considering the loss of bone mineral density. There are some limitations of the present study. Patients included in the present study had various pathological conditions possibly associated with hypovitaminosis D. Since we focused on BMI and age, which are physiological parameters potentially influenced by pathological conditions, our study could not show a direct interrelationship between vitamin D metabolism and BMI/age. However, we consider that it is meaningful to assess real-world data obtained from clinical practice in general medicine. Also, serum vitamin D levels can be affected by seasonal changes, lifestyles related to sunlight exposure, nutritional intake, and human race [5,23]. In the present study, serum vitamin D levels might have been affected by seasonal changes or sunlight exposure. All of the patients included in this study were Japanese.

Technically, although free vitamin D and albumin-bound vitamin D (10-15\%) are bioavailable, current assays cannot distinguish free vitamin D from vitamin D-binding protein-bound (DPB) vitamin D (85-90\%) and albumin-bound vitamin D, the amounts of which are affected by the capability for DPB and albumin synthesis [31]. Another limitation of this study is that it was performed retrospectively at a single center with a relatively small number of patients, and it is, therefore, difficult to draw a solid conclusion. To clarify the precise interaction between vitamin D activity and bone turnover, another study with a larger sample size including a general population or a prospective study using age- and gender-matched cohorts as a multi-center study will be needed.

In the present study, it was notable that the $1,25 \mathrm{D} / 25 \mathrm{D}$ ratio is conceivably a useful tool for suspecting bone loss, fractures, or other clinical outcomes associated with frailty.

\section{Conclusions}

The results of analysis of data for patients in our general medicine department collectively showed that increased activation of vitamin D is likely to be linked to disruption of the bone-kidney-parathyroid endocrine axis. Our findings imply the probable existence of a gender-specific difference of aging males and obese females regarding the activation of vitamin $\mathrm{D}$ that is functionally linked to bone metabolism. 


\section{Additional Information \\ Disclosures}

Human subjects: Consent was obtained or waived by all participants in this study. Ethical Committee of Okayama University Hospital issued approval KEN-2001-022. The present study was approved by the Ethical Committee of Okayama University Hospital (KEN-2001-022) and adhered to the Declaration of Helsinki. Animal subjects: All authors have confirmed that this study did not involve animal subjects or tissue. Conflicts of interest: In compliance with the ICMJE uniform disclosure form, all authors declare the following: Payment/services info: All authors have declared that no financial support was received from any organization for the submitted work. Financial relationships: All authors have declared that they have no financial relationships at present or within the previous three years with any organizations that might have an interest in the submitted work. Other relationships: All authors have declared that there are no other relationships or activities that could appear to have influenced the submitted work.

\section{References}

1. Bikle DD: Vitamin D: newer concepts of its metabolism and function at the basic and clinical level . J Endocr Soc. 2020, 4:bvz038. 10.1210/jendso/bvz038

2. Adams JS, Rafison B, Witzel S, et al.: Regulation of the extrarenal CYP27B1-hydroxylase. J Steroid Biochem Mol Biol. 2014, 144 Pt A:22-7. 10.1016/j.jsbmb.2013.12.009

3. Berger U, Wilson P, McClelland RA, Colston K, Haussler MR, Pike JW, Coombes RC: Immunocytochemical detection of 1,25-dihydroxyvitamin D3 receptor in breast cancer. Cancer Res. 1987, 47:6793-9.

4. Holick MF, Binkley NC, Bischoff-Ferrari HA, et al.: Evaluation, treatment, and prevention of vitamin D deficiency: an Endocrine Society clinical practice guideline. J Clin Endocrinol Metab. 2011, 96:1911-30. 10.1210/jc.2011-0385

5. Amrein K, Scherkl M, Hoffmann M, et al.: Vitamin D deficiency 2.0: an update on the current status worldwide. Eur J Clin Nutr. 2020, 74:1498-513. 10.1038/s41430-020-0558-y

6. Visser M, Deeg DJ, Lips P: Low vitamin D and high parathyroid hormone levels as determinants of loss of muscle strength and muscle mass (sarcopenia): the Longitudinal Aging Study Amsterdam. J Clin Endocrinol Metab. 2003, 88:5766-72. 10.1210/jc.2003-030604

7. Yoshimura N, Muraki S, Oka H, et al.: Profiles of vitamin D insufficiency and deficiency in Japanese men and women: association with biological, environmental, and nutritional factors and coexisting disorders: the ROAD study. Osteoporos Int. 2013, 24:2775-87. 10.1007/s00198-013-2372-z

8. Tamaki J, Iki M, Sato Y, et al.: Total 25-hydroxyvitamin D levels predict fracture risk: results from the 15year follow-up of the Japanese Population-based Osteoporosis (JPOS) Cohort Study. Osteoporos Int. 2017, 28:1903-13. 10.1007/s00198-017-3967-6

9. Lee P, Nair P, Eisman JA, Center JR: Vitamin D deficiency in the intensive care unit: an invisible accomplice to morbidity and mortality?. Intensive Care Med. 2009, 35:2028-32. 10.1007/s00134-009-1642-x

10. Gaksch M, Jorde R, Grimnes G, et al.: Vitamin D and mortality: individual participant data meta-analysis of standardized 25-hydroxyvitamin D in 26916 individuals from a European consortium. PLoS One. 2017, 12:e0170791. 10.1371/journal.pone.0170791

11. Martucci G, McNally D, Parekh D, et al.: Trying to identify who may benefit most from future vitamin D intervention trials: a post hoc analysis from the VITDAL-ICU study excluding the early deaths. Crit Care. 2019, 23:200. 10.1186/s13054-019-2472-z

12. Harada K, Hanayama Y, Yasuda M, et al.: Clinical relevance of low androgen to gastroesophageal reflux symptoms. Endocr J. 2018, 65:1039-47. 10.1507/endocrj.EJ18-0187

13. Ando A, Mitsuhashi T, Honda M, et al.: Risk factors for low bone mineral density determined in patients in a general practice setting. Acta Med Okayama. 2019, 73:403-11. 10.18926/AMO/57370

14. Kanda Y: Investigation of the freely available easy-to-use software 'EZR' for medical statistics . Bone Marrow Transplant. 2013, 48:452-8. 10.1038/bmt.2012.244

15. Pérez-López FR, Chedraui P, Pilz S: Vitamin D supplementation after the menopause. Ther Adv Endocrinol Metab. 2020, 11:2042018820931291. 10.1177/2042018820931291

16. Heianza Y, Arase Y, Kodama S, et al.: Effect of postmenopausal status and age at menopause on type 2 diabetes and prediabetes in Japanese individuals: Toranomon Hospital Health Management Center Study 17 (TOPICS 17). Diabetes Care. 2013, 36:4007-14. 10.2337/dc13-1048

17. Rohmer J, Hadjadj J, Bouzerara A, et al.: Serum 1,25(OH)2 vitamin D and 25(OH) vitamin D ratio for the diagnosis of sarcoidosis-related uveitis. Ocul Immunol Inflamm. 2020, 28:341-7. 10.1080/09273948.2018.1537399

18. Anderson PH: Vitamin D activity and metabolism in bone . Curr Osteoporos Rep. 2017, 15:443-9. 10.1007/s11914-017-0394-8

19. Kuro-o M: Overview of the FGF23-Klotho axis. Pediatr Nephrol. 2010, 25:583-90. 10.1007/s00467-009-12604

20. Tsujikawa H, Kurotaki Y, Fujimori T, Fukuda K, Nabeshima Y: Klotho, a gene related to a syndrome resembling human premature aging, functions in a negative regulatory circuit of vitamin $\mathrm{D}$ endocrine system. Mol Endocrinol. 2003, 17:2393-403. 10.1210/me.2003-0048

21. Shimada T, Kakitani M, Yamazaki Y, et al.: Targeted ablation of Fgf23 demonstrates an essential physiological role of FGF23 in phosphate and vitamin D metabolism. J Clin Invest. 2004, 113:561-8. 10.1172/JCI19081

22. Holick MF, Matsuoka LY, Wortsman J: Age, vitamin D, and solar ultraviolet. Lancet. 1989, 2:1104-5. 10.1016/s0140-6736(89)91124-0

23. Lanske B, Razzaque MS: Vitamin D and aging: old concepts and new insights . J Nutr Biochem. 2007, 18:7717. 10.1016/j.jnutbio.2007.02.002

24. Tuohimaa P: Vitamin D and aging. J Steroid Biochem Mol Biol. 2009, 114:78-84. 


\section{Cureus}

10.1016/j.jsbmb.2008.12.020

25. D'Andrea S, Martorella A, Coccia F, et al.: Relationship of vitamin D status with testosterone levels: a systematic review and meta-analysis. Endocrine. 2021, 72:49-61. 10.1007/s12020-020-02482-3

26. Rafiq S, Jeppesen PB: Body mass index, vitamin D, and type 2 diabetes: a systematic review and metaanalysis. Nutrients. 2018, 10:1182. 10.3390/nu10091182

27. Hajhashemy Z, Shahdadian F, Ziaei R, Saneei P: Serum vitamin D levels in relation to abdominal obesity: a systematic review and dose-response meta-analysis of epidemiologic studies. Obes Rev. 2021, 22:e13134. 10.1111/obr.13134

28. Ruiz-Ojeda FJ, Anguita-Ruiz A, Leis R, Aguilera CM: Genetic factors and molecular mechanisms of vitamin D and obesity relationship. Ann Nutr Metab. 2018, 73:89-99. 10.1159/000490669

29. Kuro-O M: Phosphate and Klotho. Kidney Int Suppl. 2011, S20-3. 10.1038/ki.2011.26

30. Nettore IC, Albano L, Ungaro P, Colao A, Macchia PE: Sunshine vitamin and thyroid. Rev Endocr Metab Disord. 2017, 18:347-54. 10.1007/s11154-017-9406-3

31. Tsuprykov O, Chen X, Hocher CF, Skoblo R, Lianghong Yin, Hocher B: Why should we measure free $25(\mathrm{OH})$ vitamin D?. J Steroid Biochem Mol Biol. 2018, 180:87-104. 10.1016/j.jsbmb.2017.11.014 